Results: 12 axSpA patients were recruited from the CASTRO cohort (42\% female, age $46 \pm 11$ years, disease duration $16 \pm 13$ years, BMI $28 \pm 5$ ). Results PRE and POST are shown in Table 1: mean values (sd), statistical significance (NS, not significant; ${ }^{*}, \mathrm{p}<0.05 ;{ }^{* \star}, \mathrm{p}<0.01$ ), and effect size. Activity indexes and CRP were lower. ASDAS and CRP shown significant differences and a large effect size. All MRI scores showed good responsiveness (ES medium-large, $p<0.01$ ), specially KITs4R. Agreement between all MRI scores were high $(r>0.8 ; p<0.01)$. Between semiautomatic and automatic methods, this agreement was also excellent $(r=0.92 ; p<0.001)$. Correlation in improvements (reductions in scores) were also significant between all MRI scores $(r>0.7 ; p<0.05)$.

\begin{tabular}{lcccc}
\hline & PRE & POST & Sign & ES \\
\hline BASDAI & $6.12(2.45)$ & $4.96(2.74)$ & N.S. & 0.44-Small \\
ASDAS & $3.61(1.04)$ & $2.58(1.25)$ & $\star *$ & 0.89 -Large \\
CRP & $11.26(8.93)$ & $4.81(4.08)$ & $* *$ & 0.84 -Large \\
BERLIN & $2.58(1.98)$ & $1.17(1.85)$ & $* *$ & $0.74-$ Medium \\
SPARCC & $3.92(3.42)$ & $1.58(2.43)$ & $* *$ & $0.69-$ Medium \\
SCAISS & $295(332)$ & $95(163)$ & $* *$ & 0.68 -Medium \\
KITS4R & $1671(1596)$ & $258(421)$ & $* *$ & 1.04 -Large \\
\hline
\end{tabular}

NS, not significant; ${ }^{*}, p<0.05 ;{ }^{* \star}, p<0.01$

Conclusion: All MRI scores have good level of agreement between them and good responsiveness. Berlin and SPARCC are observer dependent, and do not quantify the extension of the MBE area. s-SCAIS helps to this quantification. KITs4R is not observer dependent but clinimetric validation, analizing agreement level with human expert, is necessary. New advanced tools are improving quantitative and objective measurement of BME which is important to analyze responsiveness. REFERENCES:

[1] Development and validation of SCAISS, a tool for semi-automated quantification of sacroilitis by magnetic resonance in spondyloarthritis. Rheumatol Int. 2018 Oct;38(10):1919-1926.

[2] The semi-automated algorithm for the detection of bone marrow oedema lesions in patients with axial spondyloarthritis. Rheumatol Int. 2020 Apr;40(4):625-633

Disclosure of Interests: None declared.

DOI: 10.1136/annrheumdis-2021-eular.864

\section{POS0959 DIAGNOSTIC DELAY IN AXIAL SPONDYLOARTHRITIS: RESULTS FROM THE NATIONAL EARLY INFLAMMATORY ARTHRITIS AUDIT}

M. Russell ${ }^{1}$, F. Coath ${ }^{2}$, M. Yates ${ }^{1}$, K. Bechman ${ }^{1}$, S. Norton ${ }^{1}$, J. Galloway ${ }^{1}$,

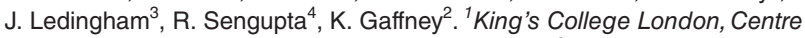
for Rheumatic Diseases, London, United Kingdom; ${ }^{2}$ Norfolk and Norwich University Hospital, Rheumatology Department, Norwich, United Kingdom; ${ }^{3}$ Portsmouth Hospitals University NHS Trust, Rheumatology Department, Portsmouth, United Kingdom; ${ }^{4}$ Royal National Hospital for Rheumatic Diseases, Department of Rheumatology, Bath, United Kingdom

Background: Diagnostic delay is a significant problem in axial spondyloarthritis (axSpA), and there is a growing body of evidence showing that delayed axSpA diagnosis is associated with worse clinical, humanistic and economic outcomes. ${ }^{1}$ International guidelines have been published to inform referral pathways and improve standards of care for patients with axSpA. ${ }^{2,3}$

Objectives: To describe the sociodemographic and clinical characteristics of newly-referred patients with axSpA in England and Wales in the National Early Inflammatory Arthritis Audit (NEIAA), with rheumatoid arthritis (RA) and mechanical back pain (MBP) as comparators.

Methods: The NEIAA captures data on all new patients over the age of 16 referred with suspected inflammatory arthritis to rheumatology departments in England and Wales. ${ }^{4}$ We describe baseline sociodemographic and clinical characteristics of axSpA patients $(n=784)$ recruited to the NEIAA between May 2018 and March 2020 , compared with $R A(n=9,270)$ and MBP $(n=370)$ during the same period.

Results: Symptom duration prior to initial rheumatology assessment was significantly longer in axSpA than RA patients $(p<0.001)$, and non-significantly longer in axSpA than MBP patients $(p=0.062): 79.7 \%$ of axSpA patients had symptom durations of $>6$ months, compared to $33.7 \%$ of RA patients and $76.0 \%$ of MBP patients; $32.6 \%$ of axSpA patients had symptom durations of $>5$ years, compared to $3.5 \%$ of RA patients and $24.6 \%$ of MBP patients (Figure $1 \mathrm{~A}$ ). Following referral, median time to initial rheumatology assessment was longer for axSpA than RA patients ( 36 vs. 24 days; $p<0.001$ ), and similar to MBP patients ( 39 days; $\mathrm{p}=0.30$ ). The proportion of axSpA patients assessed within 3 weeks of referral increased from 26.7\% in May 2018 to $34.7 \%$ in March 2020; compared to an increase from $38.2 \%$ to $54.5 \%$ for RA patients (Figure 1B). A large majority of axSpA referrals originated from primary care $(72.4 \%)$ or musculoskeletal triage services $(14.1 \%)$, with relatively few referrals from gastroenterology $(1.9 \%)$, ophthalmology $(1.4 \%)$ or dermatology $(0.4 \%)$.
Of the subset of patients with peripheral arthritis requiring EIA pathway follow-up, fewer axSpA than RA patients had disease education provided $(77.5 \%$ vs. $97.8 \%$ $p<0.001)$, and RA patients reported a better understanding of their condition $(p<0.001)$. HAQ-DI scores were lower at baseline in axSpA EIA patients than RA EIA patients ( 0.8 vs 1.1 , respectively; $p=0.004)$, whereas baseline Musculoskeletal Health Questionnaire (MSK-HQ) scores were similar (25 vs. 24, respectively; $p=0.49$ ). The burden of disease was substantial across the 14 domains compris ing MSK-HQ in both axSpA and RA (Figure 1C).

Conclusion: We have shown that diagnostic delay remains a major challenge in axSpA, despite improved disease understanding and updated referral guidelines. Patient education is an unmet need in $\operatorname{axSpA}$, highlighting the need for specialist clinics. MSK-HQ scores demonstrated that the functional impact of axSpA is no less than for RA, whereas HAQ-DI may underrepresent disability in axSpA

\section{REFERENCES:}

[1] Yi E, Ahuja A, Rajput T, George AT, Park Y. Clinical, economic, and humanistic burden associated with delayed diagnosis of axial spondyloarthritis: a systematic review. Rheumatol Ther. 2020;7:65-87.

[2] NICE. Spondyloarthritis in over 16s: diagnosis and management. 2017.

[3] van der Heijde D, Ramiro S, Landewe R, et al. 2016 update of the ASAS-EULAR management recommendations for axial spondyloarthritis. Ann Rheum Dis. 2017;76(6):978-91.

[4] British Society for Rheumatology. National Early Inflammatory Arthritis Audit (NEIAA) Second Annual Report. 2021.

A
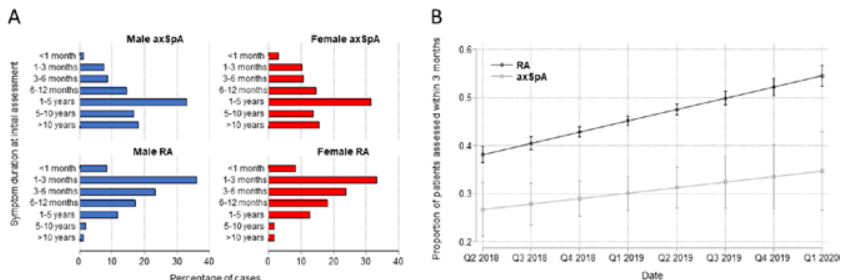

C
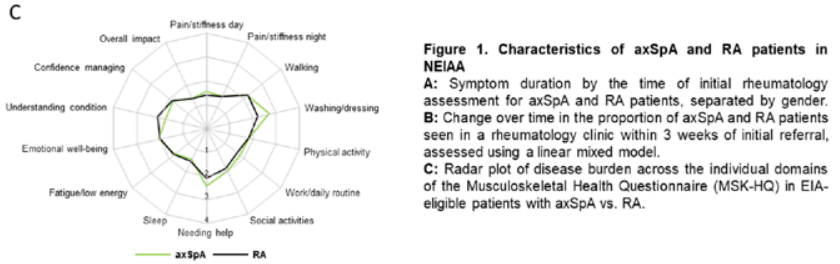

Acknowledgements: The National Early Inflammatory Arthritis Audit is commissioned by the Healthcare Quality Improvement Partnership, funded by NHS England and Improvement, and the Welsh Government, and carried out by the British Society for Rheumatology, King's College London and Net Solving.

Disclosure of Interests: Mark Russell Grant/research support from: UCB Pfizer, Fiona Coath: None declared, Mark Yates Grant/research support from UCB, Abbvie, Katie Bechman: None declared, Sam Norton: None declared James Galloway Grant/research support from: Abbvie, Celgene, Chugai, Gilead, Janssen, Lilly, Pfizer, Roche, UCB, Jo Ledingham: None declared, Raj Sengupta Grant/research support from: AbbVie, Biogen, Celgene, Lilly, MSD, Novartis, Pfizer, Roche, UCB, Karl Gaffney Grant/research support from: AbbVie, Biogen, Cellgene, Celltrion, Janssen, Lilly, Novartis, Pfizer, Roche, UCB.

DOI: 10.1136/annrheumdis-2021-eular.894

\begin{tabular}{|l}
\hline POS0960 \\
PRESENCE AND ASSOCIATED FACTORS OF \\
FATIGUE IN PATIENTS IN PATIENTS WITH AXIAL \\
SPONDYLOARTHRITIS. RESULTS FROM THE \\
EUROPEAN MAP OF AXIAL SPONDYLOARTHRITIS \\
(EMAS)
\end{tabular}

M. Garrido-Cumbrera ${ }^{1}$, V. Navarro-Compán ${ }^{2}$, L. Christen ${ }^{3}$, C. Bundy ${ }^{4}$, R. Mahapatra ${ }^{5}$, S. Makri ${ }^{6}$, C. J. Delgado-Domínguez ${ }^{1}$, J. Correa-Fernández ${ }^{1}$, S. Sanz-Gómez ${ }^{1}$, D. Poddubnyy ${ }^{7}$ on behalf of IMAS working group. ${ }^{1}$ Universidad de Sevilla, Health \& Territory Research (HTR), Seville, Spain; ${ }^{2}$ Hospital Universitario La Paz, IdiPaz, Madrid, Spain; ${ }^{3}$ Novartis Pharma AG, Patient Engagement, Basel, Switzerland; ${ }^{4}$ Cardiff University, School of Healthcare Sciences, Cardiff, United Kingdom; ${ }^{5}$ Axial Spondyloarthritis International Federation (ASIF), Patient Advocacy, London, United Kingdom; ${ }^{6}$ Cyprus League Against Rheumatism (CYPLAR), Patient Advocacy, Nicosia, Cyprus; ${ }^{7}$ Charité Universitätsmedizin Berlin, Rheumatology Department, Berlin, Germany

Background: Fatigue/tiredness is an essential aspect of disease for patients with axial spondyloarthritis (axSpA). However, little is known about its prevalence and associated factors. 
Objectives: The aim is to assess the prevalence of fatigue and associated factors in a large sample of patients with axSpA patients from 13 European countries. Methods: Data from 2,846 unselected patients of the European Map of Axial Spondyloarthritis (EMAS) through an online survey (2017-2018) across 13 European countries were analyzed.

The presence of fatigue/tiredness was evaluated using the Visual Analogue Scale from the Bath Ankylosing Spondylitis Disease Activity Index (BASDAI): "How would you describe the overall level of fatigue/tiredness you have experienced? (0-10)". Risk of poor mental health was assessed using the 12-Item General Health Questionnaire (GHQ-12; 0-12).

Possible associated factors included: Socio-demographic and disease characteristics, disease activity and function and mental health disorders.

The Mann-Whitney test was used to compare the means of variables of two categories vs. the numerical variables, the $\chi^{2}$ test was used to compare the distribution between the categorical variables. Binary logistic regression and multiple linear regression were used to identify possible predictors.

Results: A total of 2,846 axSpA patients participated in the EMAS survey: mean age was 43.9 years, $61.3 \%$ female, $48.1 \%$ had a university degree, $67.9 \%$ were married and $71.3 \%$ were HLA-B27 positive. Fatigue/tiredness was associated with younger age $(6.4 \pm 2.3$ vs $5.5 \pm 2.4)$, being female $(6.6 \pm 2.2$ vs $5.7 \pm 2.4)$, lower educational level $(6.9 \pm 2.4$ vs $6.0 \pm 2.0)$ and separated or divorced persons $(6.8 \pm 2.2$ vs $6.2 \pm 2.3$; all $p<0.001)$. Those reporting work impact $(6.8 \pm 2.1$ vs $5.8 \pm 2.4)$, physically inactive $(6.9 \pm 2.2$ vs $6.1 \pm 2.3)$ or those with sleep disorders $(7.0 \pm 2.0$ vs $5.8 \pm 2.4)$, anxiety $(7.0 \pm 2.0$ vs $5.9 \pm 2.4)$ or depression $(7.2 \pm 1.9$ vs $5.9 \pm 2.4$; all $p<0.001)$ also presented greater fatigue, as did those with higher morning stiffness $(r=0.499)$ and functional limitation ( $r=0.257)$, and poorer mental health GHQ-12 ( $r=0.419)$. Finally, the variables independently associated with fatigue were female gender $(B=0.427)$, being physical inactive $(B=-0.395)$ and those with greater morning stiffness severity $(B=0.349$; see Table 1). In addition, those on temporary and permanent sick leave, along with the unemployed, presented greater fatigue (7.1, 6.8 and 7.1 respectively).

Table 1. Linear regression analysis to predict presence of fatigue/tiredness ( $N=2052)$

\begin{tabular}{|c|c|c|c|c|c|c|}
\hline & \multicolumn{3}{|c|}{ Simple } & \multicolumn{3}{|c|}{ Multivariate } \\
\hline & B & $95 \% \mathrm{Cl}$ & p-value & B & $95 \% \mathrm{Cl}$ & p-valu \\
\hline Age & -0.018 & $-0.025,-0.011$ & $<0.001^{\star}$ & -0.015 & $-0.022,-0.008$ & $<0.001$ \\
\hline Gender (female) & 0.838 & $0.659,1.017$ & $<0.001^{*}$ & 0.427 & $0.264,0.590$ & $<0.001$ \\
\hline Marital status (married) & 0.190 & $0.042,0.339$ & $0.012^{\star}$ & 0.162 & $0.021,0.302$ & $0.024^{*}$ \\
\hline Educational level (university) & -0.274 & $-0.402,-0.146$ & $<0.001^{*}$ & -0.128 & $-0.245,-0.012$ & $0.031^{*}$ \\
\hline BMI (Overweight/Obesity) & 0.151 & $-0.026,0.328$ & 0.094 & NA & NA & NA \\
\hline $\begin{array}{l}\text { Morning stiffness severity } \\
(0-10)^{*}\end{array}$ & 0.473 & $0.442,0.505$ & $<0.001^{*}$ & 0.349 & $0.314,0.385$ & $<0.001$ \\
\hline Functional limitation (0-54) & 0.038 & $0.032,0.044$ & $<0.001^{*}$ & 0.014 & $0.008,0.019$ & $<0.001$ \\
\hline Reported Work impact (yes) & 0.936 & $0.753,1.119$ & $<0.001^{*}$ & 0.228 & $0.068,0.389$ & $0.005^{*}$ \\
\hline Physical activity (yes) & -0.726 & $-0.968,-0.485$ & $<0.001^{*}$ & -0.395 & $-0.611,-0.178$ & $<0.001^{*}$ \\
\hline Sleep disorder (yes) & 1.191 & $1.013,1.368$ & $<0.001^{*}$ & 0.276 & $0.095,0.458$ & $0.003^{*}$ \\
\hline Anxiety (yes) & 1.139 & $0.950,1.327$ & $<0.001^{*}$ & 0.002 & $-0.215,0.220$ & 0.982 \\
\hline Depres & 1.274 & $1.079,1.469$ & $<0.001^{*}$ & 0.223 & $0.001,0.446$ & $0.049^{*}$ \\
\hline GHQ-12 $(0-12)$ ** & 0.234 & $0.215,0.254$ & $<0.001$ & 0.110 & $0.088,0.132$ & $<0.001 *$ \\
\hline
\end{tabular}

${ }^{*}$ As measured by the respective item of the BASDAl scale ${ }^{\star *} 12$-item General Health Questionnaire. A value of 3 or above indicates a risk of poor mental health

Conclusion: Fatigue/tiredness was highly prevalent among axSpA European patients with female gender, engage in physical activity and those with greater morning stiffness severity most strongly associated, and the unemployed presenting greatest fatigue.

Acknowledgements: This study was supported by Novartis Pharma AG. The authors would like to thank all patients who participated in the study.

Disclosure of Interests: Marco Garrido-Cumbrera: None declared, Victoria Navarro-Compán Grant/research support from: Abbvie, BMS, Lilly, MSD, Novartis, Pfizer, Roche, and UCB, Laura Christen Employee of: Novartis Pharma AG, Christine Bundy Speakers bureau: Abbvie, Celgene, Janssen, Lilly, Novartis, and Pfizer., Raj Mahapatra: None declared, Souzi Makri: None declared, Carlos Jesús Delgado-Domínguez: None declared, José Correa-Fernández: None declared, Sergio Sanz-Gómez: None declared, Denis Poddubnyy Speakers bureau: Abbvie, BMS, Celgene, Janssen, Lilly, MSD, Novartis, Pfizer, Roche, and UCB., Grant/research support from: Abbvie, MSD, Novartis, and Pfizer. DOI: 10.1136/annrheumdis-2021-eular.968

\section{POS0961 PREVALENCE AND ASSOCIATED FACTORS OF SLEEP DISORDERS IN PATIENTS WITH AXIAL SPONDYLOARTHRITIS. RESULTS FROM THE EUROPEAN MAP OF AXIAL SPONDYLOARTHRITIS (EMAS)}

M. Garrido-Cumbrera ${ }^{1}$, V. Navarro-Compán ${ }^{2}$, L. Christen ${ }^{3}$, C. Bundy ${ }^{4}$, R. Mahapatra ${ }^{5}$, S. Makri' ${ }^{6}$, C. J. Delgado-Domínguez ${ }^{1}$, J. Correa-Fernández ${ }^{1}$,

D. Poddubnyy ${ }^{7}$ on behalf of IMAS working group. ${ }^{1}$ Universidad de Sevilla,
Health \& Territory Research (HTR), Seville, Spain; ${ }^{2}$ Hospital Universitario La Paz, IdiPaz, Madrid, Spain; ${ }^{3}$ Novartis Pharma AG, Patient Engagement, Basel, Switzerland; ${ }^{4}$ Cardiff University, School of Healthcare Sciences, Cardiff, United Kingdom; ${ }^{5}$ Axial Spondyloarthritis International Federation (ASIF), Patient Advocacy, London, United Kingdom; ${ }^{6}$ Cyprus League Against Rheumatism (CYPLAR), Patient Advocacy, Nicosia, Cyprus; ${ }^{7}$ Charité - Universitätsmedizin Berlin, Rheumatology Department, Berlin, Germany

Background: Sleep is an essential health aspect that is often impacted in patients with axial spondyloarthritis (axSpA).

Objectives: This analysis aims to assess the prevalence and associated factors of sleep disorders in a large sample of European axSpA patients.

Methods: Data were analyzed from 2,846 unselected patients with self-reported clinician-given diagnosis of axSpA of the European Map of Axial Spondyloarthritis (EMAS) through an online survey (2017-2018) across 13 European countries. Socio-demographic data; BASDAI [0-10] scores; engagement in physical activity; axSpA influence on work choice (assessed with yes/no question "Was your current or past work choice in any way determined by axSpA?"); risk of psychological distress (12-item General Health Questionnaire [GHQ-12; 0-12]); functional limitation [0-54] and self-reported anxiety and depression were evaluated. Presence of sleep disorders was assessed by the question: "Please indicate whether you have been diagnosed with any of the following: sleep disorders". A Mann-Whitney test was used to compare the means of numerical variables between dichotomous variables, the Chi-Square test was used to compare the distribution between the categorical variables. Simple and multivariable logistic regression models were used to identify associations between sleep disorders and disease characteristics, mental health and work-related variables.

Results: Age of respondents was 43.9 years; $61.3 \%$ were female; $48.1 \%$ had a university degree; $67.9 \%$ were married and $71.3 \%$ were HLA-B27 positive The prevalence of sleep disorders was $39.0 \%$. In the bivariate analysis, presence of sleep disorders was associated with female gender (68.3\% vs. $31.7 \%$; $\mathrm{p}<0.001)$; overweight/obese $(56.5 \%$ vs. $49.8 \%$; $\mathrm{p}<0.001)$; increased BASDAI scores ( $6.1 \pm 1.8$ vs. $5.0 \pm 2.1 ; p<0.001)$; fatigue $(7.0 \pm 2.0$ vs. $5.8 \pm 2.4 ; p<0.001)$ morning stiffness $(5.8 \pm 2.4$ vs. $4.8 \pm 2.4 ; p<0.001)$, work impact $(56.5 \%$ vs. $38.2 \%$ p< 0.001 ); anxiety (56.8\% vs. $12.5 \%$; $p<0.001)$; depression $(51.8 \%$ vs. $10.1 \%$; $\mathrm{p}<0.001)$ and higher GHQ-12 scores $(6.4 \pm 4.0$ vs. $3.9 \pm 3.9 ; \mathrm{p}<0.001)$. However factors that remained independently associated with sleep disorders in the multivariable analysis were anxiety $(\mathrm{OR}=3.8 \mathrm{p}<0.001)$ and depression $(\mathrm{OR}=3.1$ $\mathrm{p}<0.001)$ and female gender $(\mathrm{OR}=1.4 ; \mathrm{p}=0.002)$ [Table 1].

Table 1. Regression analysis to predict presence of sleep disorders $(\mathrm{N}=2191)$

\begin{tabular}{lcccccc}
\hline & \multicolumn{3}{c}{ Simple logistic regression } & \multicolumn{3}{c}{$\begin{array}{c}\text { Multivariable logistic } \\
\text { regression }\end{array}$} \\
\cline { 2 - 7 } & OR & $\mathbf{9 5 \%} \mathbf{~ C l}$ & p-value & OR & $\mathbf{9 5 \%}$ Cl & p-value \\
\hline Gender (female) & 1.59 & $1.36-1.87$ & $<0.001$ & 1.40 & $1.13-1.73$ & $\mathbf{0 . 0 0 2}$ \\
Marital status (married) & 1.13 & $0.99-1.28$ & 0.074 & $\mathrm{NA}$ & $\mathrm{NA}$ & $\mathrm{NA}$ \\
Overweight/Obesity & 1.31 & $1.12-1.53$ & $\mathbf{0 . 0 0 1}$ & 1.39 & $1.14-1.71$ & $\mathbf{0 . 0 0 1}$ \\
BASDAl (0-10) & 1.33 & $1.27-1.39$ & $<0.001$ & 1.07 & $0.95-1.21$ & 0.246 \\
Fatigue/Tiredness (0-10) & 1.28 & $1.23-1.33$ & $<0.001$ & 1.04 & $0.97-1.12$ & 0.271 \\
Morning Stiffness intensity (0-10) & 1.19 & $1.15-1.23$ & $<0.001$ & 1.05 & $0.98-1.13$ & 0.188 \\
Reported Work impact (yes) & 2.10 & $1.78-2.48$ & $<0.001$ & 1.29 & $1.05-1.58$ & $\mathbf{0 . 0 1 5}$ \\
Anxiety (yes) & 9.18 & $7.58-11.11$ & $<0.001$ & 3.84 & $2.99-4.94$ & $<0.001$ \\
Depression (yes) & 9.53 & $7.78-11.66$ & $<0.001$ & 3.09 & $2.37-4.02$ & $<0.001$ \\
GHQ-12 (0-12) & 1.16 & $1.14-1.19$ & $<0.001$ & 1.03 & $1.00-1.06$ & $\mathbf{0 . 0 2 9}$
\end{tabular}

${ }^{*}$ As measured by the respective item of the BASDAI scale.* 12 -item General Health Questionnaire. A value of 3 or above indicates a risk of poor mental health.

Conclusion: Sleep disorders were highly prevalent among axSpA European patients and strongly associated with female gender and reporting worse menta health, and spinal stiffness. Patients on permanent and temporary sick leave were more likely to report sleep disorders. The strong association between sleep disorders with both anxiety and depression should encourage rheumatologists to screen their patients with sleep disturbance in case they require additional specialist support.

Acknowledgements: This study was supported by Novartis Pharma AG. The authors would like to thank all patients who participated in the study.

Disclosure of Interests: Marco Garrido-Cumbrera: None declared, Victoria Navarro-Compán Grant/research support from: Abbvie, BMS, Lilly, MSD, Novartis, Pfizer, Roche, and UCB., Laura Christen Employee of: Novartis Pharma AG, Christine Bundy Speakers bureau: Abbvie, Celgene, Janssen, Lilly, Novartis, and Pfizer, Raj Mahapatra: None declared, Souzi Makri: None declared, Carlos Jesús Delgado-Domínguez: None declared, José Correa-Fernández: None declared, Denis Poddubnyy Speakers bureau: Abbvie, BMS, Celgene, Janssen, Lilly, MSD, Novartis, Pfizer, Roche, and UCB, Grant/research support from: Abbvie, MSD, Novartis, and Pfizer.

DOI: 10.1136/annrheumdis-2021-eular.981 